\title{
Os desafios dos enfermeiros para realização do Pré-Natal na Estratégia de Saúde da Família
}

\author{
The nurses' challenges for performing Prenatal Care in the Family Health Strategy \\ Los desafíos de las enfermeras para la realización del cuidado Prenatal En la Estrategia de Salud de \\ la Familia
}

\section{Vitória da Silva Gomes de Melo ${ }^{1 *}$, Elisângela do Nascimento Fernandes Gomes ${ }^{2}$, Eliara Adelino da Silva $^{3}$, Lília Marques Simões Rodrigues ${ }^{4}$, Geisa Sereno Velloso da Silva ${ }^{5}$, Cátia Maria Santos Diogo da Silva ${ }^{6}$.}

Como citar esse artigo. de Melo, VSG; Gomes, ENF; da Silva, EA; Rodrigues, LMS; da Silva, GSV; da Silva, CMSD. Os desafios dos enfermeiros para realização do Pré-Natal na Estratégia de Saúde da Família. Revista PróUniverSUS. 2021 Jul./Dez.; 12 (2) SUPLEMENTO: 02-09.

\section{Resumo}

O presente estudo tem como objetivos identificar e analisar as dificuldades que os enfermeiros enfrentam para realizar uma assistência de enfermagem no pré-natal nas Estratégias de Saúde da Família e identificar quais as estratégias utilizadas nas Estratégias da Família para o pré-natal. Metodologia: Trata-se de uma revisão da literatura, de caráter descritivo e exploratório, com abordagem qualitativa. Através da revisão de literatura possibilita colocar o pesquisador em contato direto com tudo o que foi escrito ou dito sobre determinado assunto. O método deste estudo é o qualitativo, responde questões muito particulares, seu foco é de caráter subjetivo do objetivo analisado, trabalha aspectos da realidade que não são quantificados, busca compreensão das relações sociais. Resultados: Os desafios identificados nas pesquisas enfrentadas pelos enfermeiros para realização do prénatal foram: a deficiência do acolhimento das mulheres, as fragilidades na qualificação profissional da equipe, a precariedade de recursos relativos a área física, recursos humanos e materiais nas unidades de saúde, o desconhecimento da comunidade sobre as atribuições do exercício profissional e da consulta de enfermagem. A principais estratégias para realização do pré-natal foram: busca ativa das gestantes, grupo de gestantes, acolhimento humanizado da gestante e família e atualização da equipe. Conclusão: É evidente a necessidade da capacitação profissional, a conscientização da comunidade sobre as atribuições e a realização da consulta de pré-natal feita pelo enfermeiro, para ampliação da adesão das gestantes ao pré-natal, e que o mesmo seja satisfatório e de qualidade.

Palavras-chave: Enfermagem; Pré-Natal; Saúde da Mulher.

\begin{abstract}
The present study aims to identify and analyze the difficulties that nurses face to perform nursing care in prenatal care in the Family Health Strategies and to identify which strategies are used in the Family Strategies for prenatal care. Methodology: This is a literature review, of a descriptive and exploratory nature, with a qualitative approach. Through the literature review, it makes it possible to put the researcher in direct contact with everything that has been written or said on a given subject. The method of this study is qualitative, it answers very particular questions, its focus is subjective in nature of the analyzed objective, it works aspects of reality that are not quantified, it seeks to understand social relations. Results: The challenges identified in the researches faced by nurses to perform prenatal care were: the deficiency in the reception of women, the weaknesses in the professional qualification of the team, the precariousness of resources related to the physical area, human and material resources in the health units, the community's lack of knowledge about the attributions of professional practice and nursing consultation. The main strategies for carrying out prenatal care were: active search of pregnant women, group of pregnant women, humanized reception of pregnant women and family and updating of the team. Conclusion: It is evident the need for professional training, community awareness about the duties and the performance of the prenatal consultation made by the nurse, to increase the adherence of pregnant women to prenatal care, and that it is satisfactory and of quality.

Keywords: Nursing; Prenatal; Women's Health.
\end{abstract}

$1^{*}$ Acadêmica do Curso de Enfermagem, Universidade de Vassouras. RJ. Brasil. ORCID: https://orcid.org/0000-0002-9987-9419.

${ }^{2}$ Mestre em Ciências Ambientais pela Universidade de Vassouras. Docente do Curso de Enfermagem da Universidade de Vassouras. RJ. Brasil. ORCID: http://orcid.org/0000-0001$8432-4157$.

${ }^{3}$ Mestre em Ciências da Saúde e Meio Ambiente. Docente do Curso de Enfermagem da Universidade de Vassouras. RJ. Brasil. ORCID: https://orcid.org/0000-0003-4387-6662. ${ }^{4}$ Mestre em Enfermagem pela EEAAC-UFF. MBA em Administração Hospitalar, Coordenadora do Curso de Enfermagem da Universidade de Vassouras, Vassouras, RJ, Brasil. ORCID: https://orcid.org/0000-0003-2979-6316.

${ }^{5}$ Mestre em Enfermagem pela UFRJ. Docente do Curso de Enfermagem, Universidade de Vassouras. RJ. Brasil. ORCID: https://orcid.org/0000-0003.0304-8010.

${ }^{6}$ Doutora. Docente do Curso de Enfermagem da Universidade de Vassouras, Vassouras, RJ, Brasil. ORCID: https://orcid.org/0000-0002-7000-5961

* Email de correspondencia: vitoriiagomes@hotmail.com 


\section{Resumen}

El presente estudio tiene como objetivo identificar y analizar las dificultades que enfrentan las enfermeras para realizar el cuidado de enfermería en la atención prenatal en las Estrategias de Salud de la Familia e identificar qué estrategias se utilizan en las Estrategias Familiares para la atención prenatal. Metodología: Se trata de una revisión de la literatura, de carácter descriptivo y exploratorio, con enfoque cualitativo. A través de la revisión de la literatura, permite poner al investigador en contacto directo con todo lo que se ha escrito o dicho sobre un tema determinado. El método de este estudio es cualitativo, responde a preguntas muy particulares, su enfoque es subjetivo en el objetivo analizado, trabaja aspectos de la realidad que no están cuantificados, busca comprender las relaciones sociales. Resultados: Los desafíos identificados en las investigaciones que enfrentan las enfermeras para realizar la atención prenatal fueron: la deficiencia en la recepción de mujeres, las debilidades en la calificación profesional del equipo, la precariedad de recursos relacionados con el área física, recursos humanos y materiales en las unidades de salud., el desconocimiento de la comunidad sobre las atribuciones de la práctica profesional y la consulta de enfermería. Las principales estrategias para la realización de la atención prenatal fueron: búsqueda activa de gestantes, grupo de gestantes, acogida humanizada de gestantes y familiares y actualización del equipo. Conclusión: Es evidente la necesidad de formación profesional, conciencia comunitaria sobre los deberes y el desempeño de la consulta prenatal realizada por la enfermera, para incrementar la adherencia de las gestantes a la atención prenatal, y que sea satisfactoria y de calidad.

Palabras clave: Enfermería; Prenatal; La salud de la mujer.

\section{Introdução}

A gestação é uma vivência complexa e singular para cada mulher, pois é nessa fase que as alterações biológicas, fisiológicas e emocionais acontecem. Com isso, a assistência a mulher no Pré-Natal é coordenada por políticas públicas com o objetivo de prestar uma assistência de qualidade e humanizada, reduzir o número de morbimortalidade materna e neonatal. ${ }^{1}$

O Ministério da Saúde define o pré-natal como um período antes do nascimento da criança onde são realizadas ações para promoção do bem-estar materno e infantil tanto de modo individual como de modo coletivo já que nesse período as gestantes devem ser acompanhadas e assistidas de modo integral para que sua gravidez seja saudável, realizando toda rotina recomendada no pré-natal, como os exames clínicos, laboratoriais, orientações por parte dos profissionais de saúde para promoção do auto cuidado no ciclo gravídico e puerperal. A consulta de pré-natal deve ser iniciada no primeiro trimestre da gestação, a mulher deve realizar no mínimo de 6 consultas, uma no primeiro trimestre, duas no segundo trimestre e três no terceiro trimestre, sendo de extrema importância orientações e esclarecimento de dúvidas e o envolvimento das equipes para que o cuidado seja satisfatório tanto para mãe quanto para a própria equipe. $^{2}$

A assistência ao Pré-Natal é oferecida na Estratégia de Saúde da Família que por ser próximo das residências dos usuários gera um vínculo, com atendimento humanizado e implementação de ações individuais e coletivas para a promoção e prevenção da qualidade de vida da população. ${ }^{3}$

Conforme a Lei 7.498/86 de 24 de Julho de 1986, o profissional enfermeiro tem respaldo legal, técnico, científico e total autonomia para realizar a assistência ao pré-natal nas Estratégias de Saúde da Família, promover a saúde, prevenção de doenças e desfrutar da humanização no cuidado prestado, elaborando um plano de assistência de enfermagem na consulta de prénatal, de acordo com as reais necessidades da gestante, intervindo, orientando e direcionando a outros serviços se necessário, proporcionando a interdisciplinaridade das ações. ${ }^{4}$

De acordo com resultados de vários estudos científicos, a assistência ao pré-natal no Brasil, apresenta várias fragilidades nos serviços para o atendimento da mulher e parceiro durante o ciclo gravídico e puerperal, o que acaba interferindo de forma direta e indiretamente na qualidade da assistência. Muitas das vezes o prénatal não é realizado, ou é feito de forma inadequada, que se resulta com baixa efetuação da avaliação clínica, redução na realização dos exames, ausência de diagnóstico e tratamento precoce de infecções e patologias, contribuindo para ocorrências de diversos problemas: entre eles a prematuridade e complicações maternas, favorecendo o aumento dos índices de morbimortalidade materna e infantil no Brasil. ${ }^{5}$

Os profissionais enfermeiros enfrentam dificuldades para adesão e captação precoce das gestantes para a realização do pré-natal e para esses desafios/dificuldades é necessário a criação de estratégias para uma melhor assistência nesse período de grande importância a mulher. ${ }^{5}$

O objetivo desse estudo é identificar e analisar as dificuldades que os enfermeiros enfrentam para realizar uma assistência de enfermagem no pré-natal de baixo risco nas Estratégias de Saúde da Família e identificar quais as estratégias utilizadas nas Estratégias da Família para o pré-natal de baixo risco.

\section{Metodologia}

Trata-se de uma revisão de literatura de caráter exploratório, a respeito dos desafios enfrentados pelos enfermeiros para realização do pré-natal de baixo risco nas Estratégias de Saúde da Família (ESF). A 
pesquisa exploratória tem como objetivo desenvolver, esclarecer e modificar conceitos e ideias, tendo em vista a formulação de problemas mais precisos ou hipóteses pesquisáveis para estudos posteriores. ${ }^{6}$

A revisão de literatura tem como finalidade colocar o pesquisador em contato direto com tudo o que foi escrito ou dito sobre determinado assunto. ${ }^{7}$

O método deste estudo é qualitativo, que de acordo com a literatura, responde a questões muito particulares, seu foco é de caráter subjetivo do objetivo analisado. Ela se preocupa, nas ciências sociais, com um nível de realidade que não pode ser quantificado. ${ }^{8}$

Os dados foram coletados em base de dados virtuais. Para tal utilizou-se a Biblioteca Virtual de Saúde (BVS), na seguinte base de informação: Biblioteca Regional de Medicina (BIREME); Literatura LatinoAmericana e do Caribe em Ciências da Saúde (LILACS), Scientific Eletronic Library Online (SCIELO), dentre outros, no período de agosto à novembro de 2020.

Optou-se pelos seguintes descritores: Enfermagem; Pré-Natal; Saúde da Mulher.

Estabeleceu-se então para a realização da pesquisa os critérios de inclusão: textos na íntegra e em português com abordagem da temática estabelecida e que obedecessem ao recorte temporal de 2013 a 2020 e como critérios de exclusão, os textos incompletos e em língua estrangeira, textos que não abordassem a temática estabelecida e com recorte temporal inferior a 2013

Após a associação de todos os descritores foram encontrados 18 artigos e selecionados apenas 10 artigos.

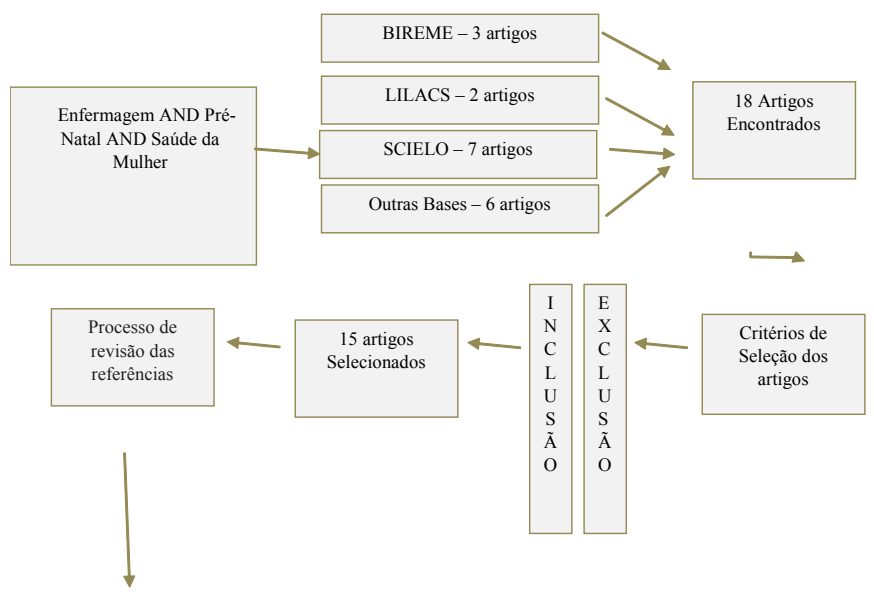

10 Artigos Selecionado

Figura 1. Fluxograma das referências selecionadas.

\section{Resultados e Discussão}

Dez estudos preencheram os critérios de elegibilidade e foram incluídos nesta revisão. $\mathrm{O}$ quadro 1, a seguir, apresenta a caracterização dos estudos levando-se em consideração o título, país e ano da publicação, objetivo, principais resultados e tipo de estudo.

Entre os dez artigos analisados identificou-se que todos foram realizados no Brasil e publicados no idioma português. Destacou-se o ano de 2019 com quatro artigos publicados. Quanto ao tipo de estudo, evidenciou-se um estudo qualitativo exploratório descritivo, uma revisão narrativa de literatura, três estudo descritivo qualitativo, duas revisão integrativa de literatura, um estudo descritivo de abordagem quali-quantitativa, um estudo descritivo exploratório e uma revisão sistemática.

Para análise dos dados, os estudos foram agrupados em duas categorias de análise: Fatores que dificultam a realização do Pré-Natal de Baixo Risco do enfermeiro na Estratégia de Saúde da Família e Estratégias utilizadas nas Estratégias de Saúde da Família para o Pré-Natal de Baixo Risco.

\section{Fatores que dificultam ou influenciam a realização do pré-natal pelos enfermeiros}

Dos sete artigos incluídos nesta categoria todos evidenciaram a dificuldade dos enfermeiros na realização do pré-natal de baixo risco. Dentre os fatores citados que dificultaram ou influenciaram a realização do pré-natal de baixo risco pelos enfermeiros estão: falta de acolhimento das gestantes por parte dos profissionais, a precariedade de recursos relativos a área física, recursos humanos e materiais, sobrecarga de atividades propostas ao enfermeiro, o desconhecimento do trabalho do enfermeiro e da consulta de enfermagem e a necessidade de maior qualificação profissional.

A falta de acolhimento e pouca prestatividade por parte dos profissionais enfermeiros em atender aos questionamentos feitos pelas gestantes no prénatal, destaca-se sendo uma das atitude que dificulta a adesão das mesmas na consulta de pré-natal, pela falta de interesse e responsabilidade pelas suas necessidades trazendo com isso, sentimentos de insegurança, desinformação, desamparo, desproteção, desrespeito e desprezo, pois através do acolhimento da gestante, que o profissional enfermeiro cria um vínculo, deixando a gestante mais a vontade para sanar suas dúvidas, utilizando a escuta qualificada, sem julgamentos ou preconceitos, garantindo a confidencialidade e privacidade, orientando quanto aos cuidados durante a gestação e incentivando a presença do acompanhante durante as consultas de pré-natal. Sem essas atitudes por parte dos profissionais, a consulta de pré-natal acaba 
Quadro 1. Caracterização dos artigos primários incluídos no estudo.

\begin{tabular}{|c|c|c|c|c|}
\hline Título & País/Ano & Objetivo & Principais Resultados & Tipos de Estudo \\
\hline $\begin{array}{l}\text { Consulta de En- } \\
\text { fermagem no Pré- } \\
\text { Natal: Narrativas } \\
\text { de Gestantes e En- } \\
\text { fermeiras }\end{array}$ & Brasil 2019 & $\begin{array}{l}\text { Analisar a consulta de en- } \\
\text { fermagem no Pré-Natal, } \\
\text { a partir da perspectiva de } \\
\text { gestantes e enfermeiras }\end{array}$ & $\begin{array}{l}\text { Identificou-se que as gestantes expressaram sa- } \\
\text { tisfação com o exame físico, destacando o aco- } \\
\text { lhimento, havendo queixas quanto a competên- } \\
\text { cia técnica das enfermeiras especificamente em } \\
\text { aconselhamento de infecção urinária, falta de } \\
\text { alguns medicamentos prescritos e prazos longos } \\
\text { para realizar e receber exames de natureza pre- } \\
\text { ventiva. }\end{array}$ & $\begin{array}{l}\text { Qualitativo, explora- } \\
\text { tório e descritivo }\end{array}$ \\
\hline $\begin{array}{l}\text { Atuação do Enfer- } \\
\text { meiro na Consulta } \\
\text { Pré-Natal: Limites } \\
\text { e Potencialidades }\end{array}$ & Brasil 2016 & $\begin{array}{l}\text { Identificar os limites e as } \\
\text { potencialidades da atuação } \\
\text { do enfermeiro na consulta } \\
\text { Pré-Natal }\end{array}$ & $\begin{array}{l}\text { Os limites destacados foram atuação do enfer- } \\
\text { meiro no pré-natal, atenção baseada no modelo } \\
\text { biomédico, precariedade de recursos e o desco- } \\
\text { nhecimento do trabalho do enfermeiro. As poten- } \\
\text { cialidades destacadas foram acolhimento, víncu- } \\
\text { lo e interação, prática de educação em saúde e o } \\
\text { comprometimento do profissional enfermeiro }\end{array}$ & $\begin{array}{c}\text { Revisão Narrativa de } \\
\text { Literatura }\end{array}$ \\
\hline $\begin{array}{l}\text { Assistência ao Pré- } \\
\text { Natal: Depoimen- } \\
\text { tos de Enfermeiras }\end{array}$ & Brasil 2018 & $\begin{array}{l}\text { Avaliar a assistência ao } \\
\text { pré-natal de bauxo risco } \\
\text { realizada pelo enfermeiro } \\
\text { no município de Lagarto/ } \\
\text { SE }\end{array}$ & $\begin{array}{l}\text { Evidenciou-se que o pré-natal de baixo risco no } \\
\text { município de Lagarto, realizado pelos enfermei- } \\
\text { ros é feito de forma satisfatória, sendo que ainda } \\
\text { há ações de enfermagem como anamnese e exa- } \\
\text { me físico são realizados de maneira superficial } \\
\text { devido a grande demanda da ESF, além de não } \\
\text { haver captação precoce das gestantes de forma } \\
\text { satisfatória e também a pouca valorização da } \\
\text { educação em saúde e de promoção e prevenção } \\
\text { no âmbito da assistência ao pré-natal }\end{array}$ & $\begin{array}{l}\text { Descritivo qualita- } \\
\text { tivo }\end{array}$ \\
\hline $\begin{array}{l}\text { Pré-Natal da Ges- } \\
\text { tante de Risco } \\
\text { Habitual: Poten- } \\
\text { cialidades e Fragi- } \\
\text { lidades }\end{array}$ & Brasil 2019 & $\begin{array}{l}\text { Conhecer as potenciali- } \\
\text { dades e fragilidades na } \\
\text { consulta pré-natal de risco } \\
\text { habitual }\end{array}$ & $\begin{array}{l}\text { Tivemos o acolhimenro, o fácil acesso, a reali- } \\
\text { zação de exames e os grupos de gestantes como } \\
\text { potencialidades do pré-natal e como fragilidades, } \\
\text { tivemos a falta de organização das unidades, de } \\
\text { medicamentos de uso essencial, de recursos ma- } \\
\text { teriais, o tempo prolongado para início das con- } \\
\text { sultas e para as realizações dos exames }\end{array}$ & $\begin{array}{c}\text { Descritivo qualita- } \\
\text { tivo }\end{array}$ \\
\hline $\begin{array}{l}\text { Desafios do Enfer- } \\
\text { meiro no Pré-Natal } \\
\text { de Baixo Risco }\end{array}$ & Brasil 2020 & $\begin{array}{l}\text { Identificar os desafios do } \\
\text { enfermeiro na realização } \\
\text { do pré-natal de baixo risco }\end{array}$ & $\begin{array}{l}\text { Identificou-se a importância do enfermeiro na } \\
\text { atenção ao pré-natal, através das ações desen- } \\
\text { volvidas na consulta de enfermagem, e mesmo } \\
\text { diante da sobrecarga de atribuições, o enfermeiro } \\
\text { realiza acolhimento e escuta qualificada para di- } \\
\text { recionar as ações assistenciais e também algumas } \\
\text { situações limitadoras na assistência prestada pelo } \\
\text { enfermeiro, como estrutura física inadequada, so- } \\
\text { brecarga de atribuições e ausência de vinculação } \\
\text { entre a gestante e a maternidade de referência }\end{array}$ & $\begin{array}{l}\text { Revisão integrativa } \\
\text { de literatura }\end{array}$ \\
\hline $\begin{array}{l}\text { Captação da Ges- } \\
\text { tante para o Pré- } \\
\text { Natal Precoce }\end{array}$ & Brasil 2020 & $\begin{array}{l}\text { Identificar as dificuldades } \\
\text { do enfermeiro da Atenção } \\
\text { Básica para captação de } \\
\text { gestantes no pré-natal }\end{array}$ & $\begin{array}{l}\text { Percebeu-se que mais do que dificuldades opera- } \\
\text { cionais ou de contingência, a grande dificuldade } \\
\text { que se apresenta aos enfermeiros na atenção bá- } \\
\text { sica em relação ao primeiro trimestre gestacio- } \\
\text { nal é relacionada aos baixos índices de captação } \\
\text { de gestantes para a assistência ao pré-natal, di- } \\
\text { ficuldades sobretudo na captação precoce e em } \\
\text { relação a dificuldade dos enfermeiros foi possível } \\
\text { constatar que muitos desses profissionais têm di- } \\
\text { ficuldades inclusive de compreensão dos termos } \\
\text { e da política de saúde e das estratégias do país em } \\
\text { relação a diminuição dos índices de mortalidade } \\
\text { e de aumento da captação no pré-natal }\end{array}$ & $\begin{array}{l}\text { Descritivo quali- } \\
\text { quantitativo }\end{array}$ \\
\hline $\begin{array}{l}\text { Características do } \\
\text { Atendimento Pré- } \\
\text { Natal na Rede Bá- } \\
\text { sica de Saúde }\end{array}$ & Brasil 2013 & $\begin{array}{l}\text { Analisar as características } \\
\text { do atendimento pré-natal } \\
\text { na rede de atenção básica } \\
\text { a saúde }\end{array}$ & $\begin{array}{l}\text { Evidenciou-se que existem inadequações relacio- } \\
\text { nadas ao início tardio do pré-natal, a realização } \\
\text { da USG e a baixa participação das gestantes em } \\
\text { atividades educativas }\end{array}$ & $\begin{array}{l}\text { Descritivo explora- } \\
\text { tório }\end{array}$ \\
\hline
\end{tabular}


Quadro 1 (cont.). Caracterização dos artigos primários incluídos no estudo.

\begin{tabular}{|c|c|c|c|c|}
\hline Título & País/Ano & Objetivo & Principais Resultados & Tipos de Estudo \\
\hline $\begin{array}{l}\text { Impacto de Estraté- } \\
\text { gias Educacionais } \\
\text { no Pré-Natal de } \\
\text { Baixo Risco }\end{array}$ & Brasil 2016 & $\begin{array}{l}\text { Analisar a partir de uma } \\
\text { revisão sistemática o im- } \\
\text { pacto de estratégias edu- } \\
\text { cacionais desenvolvidas } \\
\text { no pré-natal de baixo risco } \\
\text { em resultados obstétricos }\end{array}$ & $\begin{array}{l}\text { Observou-se que as estratégias de intervenção } \\
\text { desenvolvidas no pré-natal, com grupos de ges- } \\
\text { tantes e nas visitas domiciliares por profissionais } \\
\text { e pessoas residentes nas áreas de moradia das } \\
\text { mulheres, construídas e desenvolvidas de forma } \\
\text { pedagógica e participativa, contribuiram de modo } \\
\text { significativo para redução da prematuridade e do } \\
\text { baixo peso ao nascer e aumento da prevalência de } \\
\text { aleitamento materno exclusivo }\end{array}$ & Revisão sistemática \\
\hline $\begin{array}{l}\text { Acolhimento Hu- } \\
\text { manizado no Cui- } \\
\text { dado Pré-Natal as } \\
\text { Gestantes da ESF }\end{array}$ & Brasil 2019 & $\begin{array}{l}\text { Apresentar os procedi- } \\
\text { mentos a serem executa- } \\
\text { dos no acolhimento, que } \\
\text { sejam capazes de assegu- } \\
\text { rar as gestantes atendidas } \\
\text { pela ESF, um pré-natal de } \\
\text { qualidade }\end{array}$ & $\begin{array}{l}\text { Apesar dos esforços do Ministério da Saúde, } \\
\text { que incluem a construção da Rede Cegonha em } \\
\text { 2011, muito precisa ser feito para que a redução } \\
\text { da mortalidade materna e neonatal se concretize } \\
\text { como por exemplo facilitar o acesso ao programa } \\
\text { pré-natal; prestar um atendimento humanizado e } \\
\text { contínuo com a utilização de tecnologias leves, } \\
\text { intervindo no que for necessário; fornecer orien- } \\
\text { tações e informações que envolvem a mãe e o } \\
\text { bebê; realizar consultas de modo a deixar a ges- } \\
\text { tante a vontade para fazer perguntas, procurando } \\
\text { respondê-las }\end{array}$ & $\begin{array}{c}\text { Revisão integrativa } \\
\text { de literatura }\end{array}$ \\
\hline $\begin{array}{l}\text { Consulta de Pré- } \\
\text { Natal na Atenção } \\
\text { Primária a Saú- } \\
\text { de: Fragilidades } \\
\text { e Potencialidades } \\
\text { da Intervenção de } \\
\text { Enfermeiros Brasi- } \\
\text { leiros }\end{array}$ & Brasil 2020 & $\begin{array}{l}\text { Conhecer as fragilidades } \\
\text { e potencialidades da inter- } \\
\text { venção do enfermeiro na } \\
\text { consulta de pré-natal }\end{array}$ & $\begin{array}{l}\text { Como fragilidades, a morosidade na entrega dos } \\
\text { exames solicitados no pré-natal, o déficit de pro- } \\
\text { fissionais para compor as equipes multiprofissio- } \\
\text { nais e a dificuldade no atendimento das gestantes } \\
\text { acerca da importância do pré-natal e como poten- } \\
\text { cialidades, a variedade de intervenções clínicas, } \\
\text { o vínculo entre o profissional e a gestante e o uso } \\
\text { de protocolos municipais }\end{array}$ & $\begin{array}{c}\text { Descritivo qualita- } \\
\text { tivo }\end{array}$ \\
\hline
\end{tabular}

se tornando algo insatisfastório e em consequência disso, afasta a gestante desse ambiente de cuidado de extrema importância para o momento que a mesma está vivenciando. ${ }^{9-10}$

Outro ponto que dificulta, é em relação a precariedade de recursos relativos a área física, materiais e humanos. A ausência de um espaço físico apropriado para a realização das consultas de pré-natal e atividades em grupo voltadas para a educação em saúde das gestantes, interfere na qualidade da assistência e prejudica a privacidade da gestante. Preservar um ambiente adequado ao inter-relacionamento entre a equipe de saúde e a gestante destinado a assistência ao pré-natal, deve ser garantido, pois é essencial para que a consulta seja de qualidade e a criação de vínculo seja efetiva, esclarecendo dúvidas, permitindo que a gestante exponha seus medos e ansiedade.

O enfermeiro que atua na ESF, tem uma demanda ampla de atribuições e funções, tanto administrativas como assistenciais em diversos programas que são inseridos na unidade de Saúde. Observa-se o esgotamento do profissional com o excesso de tarefas, o que afeta diretamente na assistência a saúde da mulher no período gestacional, pois o atendimento da mesma, não se restringe somente a consulta de pré-natal, o enfermeiro deve realizar visita domiciliar, busca ativa para o prénatal precoce, busca ativa das gestantes faltosas e seus parceiros, atividades educativas entre outras atividades relacionadas a saúde materna. Fica claro a necessidade de ampliar o número de enfermeiros nas ESF, a sobrecarga das atividades propostas ao enfermeiro, limita a excelência de seu trabalho. ${ }^{1-9-10-11-12}$

O desconhecimento do trabalho do enfermeiro e da consulta de enfermagem é outra difuldade que interfere negativamente para a adesão da consulta de prénatal, realizada pelo enfermeiro. A falta de conhecimento da população quanto as atribuições, habilidades e competências do profissional de enfermagem é algo que merece ser discutido. As competências do enfermeiro precisam ser mais trabalhadas na comunidade, o profissional necessita demonstrar conhecimento, capacidade de resolução e empatia no atendimento ao usuário. $^{9}$

A maioria das gestantes entendem que a consulta de pré-natal é uma atribuição específica do médico e que o enfermeiro só auxilia se caso houver necessidade. $\mathrm{O}$ enfermeiro para elas só tem como função a verificação dos sinais vitais, mensuração do peso e altura e vacinação. Isso se dá devido aos aspectos históricos em que os enfermeiros são vistos como profissionais 
incapacitados para a realização do atendimento e acompanhamento integral de gestantes de baixo risco, sendo evidente o desconhecimento da população em geral e das mulheres em particular em relação ao saber fazer científico do profissional enfermeiro, capacitado para a realização da enfermagem no pré-natal. É previsto na Lei 7.498/86 de 24 de Julho de 1986, que o profissional enfermeiro tem total autonomia para realizar as consultas de pré-natal ficando responsável de promover a saúde, prevenção de doenças e desfrutar da humanização no cuidado prestado, elaborando um plano de assistência de enfermagem na consulta de prénatal, de acordo com as reais necessidades da gestante, intervindo, orientando e direcionando a outros serviços se necessário, proporcionando a interdisciplinaridade das ações. ${ }^{9}$

Quanto a qualificação profissional por parte dos enfermeiros, é necessário que os mesmos invistam mais na sua área específica por meio de capacitações, conscientizando as equipes sobre essa necessidade e importância da educação em saúde para a promoção do cuidado, pois é visível que alguns enfermeiros ainda apresentam dificuldades para realizar procedimentos que exigem conhecimento e habilidades e isso também é um ponto que acaba dificultando a consulta de prénatal. Frequentemente, com a correria da rotina do dia-a-dia do trabalho muitos acabam deixando de lado algumas práticas essenciais para a realização do cuidado, prejudicando a qualidade e eficiência no atendimento prestado, por isso, a educação continuada e permanente deve tornar-se uma realidade no cotidiano do exercício da profissão. ${ }^{9-13}$

\section{Estratégias utilizadas pelos enfermeiros para realização do pré-natal}

Foram identificados 3 artigos que evidenciaram estratégias realizadas pelos enfermeiros para que a realização da consulta de pré-natal seja de qualidade e efetiva. As estratégias são: $\mathrm{O}$ acolhimento humanizado da gestante e família; busca ativa das gestantes, grupo de gestantes para sanar as dúvidas e orientar quanto aos cuidados na gestação e atualização da equipe de forma contínua para prestar um melhor atendimento.

O acolhimento é uma das principais estratégias para facilitar o acesso e o vínculo do usuário a Estratégia de Saúde da Família (ESF), deve ser realizadas por todos da equipe, deve ser feita desde a chegada do usuário até a sua saída, deixando-o a vontade para expor suas queixas e dúvidas, levando em conta suas preocupações e angústias e com isso, utilizando uma escuta qualificada para que se garanta uma atenção integral, resolutiva e responsável. ${ }^{2}$

As gestantes procuram um atendimento humanizado, sem intervenções desnecessárias, que as faça sentir valorizadas e seguras, o que inclui o olhar e o toque. Oprofissional enfermeiroé o principal responsável para recepcionar a gestante durante o atendimento, atuando no oferecimento da atenção integralizada para a resolução da continuação da assistência à saúde. ${ }^{14-15}$

A busca ativa é usada para captação precoce das gestantes e para captação das gestantes faltosas, esta atividade é realizada pelos enfermeiros e Agentes Comunitários de Saúde (ACS) através de visitas domiciliares, onde os mesmos identificam os motivos no qual as gestantes não comparecem a ESF para consulta de pré-natal, neste momento o profissional deve orientar e conscientizar a mulher, seu parceiro e família, sobre a importância da assistência a saúde no período gestacional. O ínicio do pré-natal no primeiro trimestre de gestação e adesão da mulher as consultas, é essencial, é um indicador de qualidade da assistência ao Pré-natal. A mulher irá conseguir realizar todas as rotinas de pré-natal que são recomendadas pelo Ministério da Saúde. Terá a oportunidade de fazer todas as ações preventivas, diagnósticos e promoção a saúde e além disso, facilitar a identificação de situações de alto risco que requerem encaminhamentos imediatos para serviços de saúde de alta complexidade para melhor planejamento do cuidado. ${ }^{1-14}$

É muito importante que a gravidez seja diagnosticada precocemente, para que a assistência ao pré-natal não comece após a $12^{\mathrm{a}}$ semana de gestação, pois isso piora os resultados em relação a morbimortalidade materno-infantil. A busca ativa deve ser uma das estratégias/ferramentas utilizadas pela equipe de saúde, uma vez que identifica as situações de risco, acompanhando as gestantes e fortalecendo cada vez mais o vínculo entre a equipe e a gestante. ${ }^{1-14}$

O grupo de gestantes é uma reunião que geralmente acontece em forma de roda de conversa, onde o enfermeiro tem a função de complementar o conhecimento próprio das gestantes, esclarecer suas dúvidas e com isso criar um vínculo entre eles. A dinâmica de grupo favorece a troca de experiências e ajuda a desfazer o ciclo de ansiedades e temor entre as gestantes. ${ }^{2}$

Uma estratégia que também é utilizada pelos enfermeiros no pré-natal é o grupo de gestantes que possibilita o iintercâmbio de experiências e conhecimentos, permitindo que as mesmas fiquem a vontade para esclarecer suas dúvidas relacionadas a gestação e em consequência disso fortalece a adesão ao pré-natal. O grupo de gestantes quando desenvolvido de modo dinâmico estimula a participação das gestantes no pré-natal e contribui para redução do risco de prematuridade, além de aumentar as taxas do aleitamento materno exclusivo. ${ }^{15}$

O conhecimento na área da saúde está em constante mudança e é imprescindível que o profissional da área esteja alinhado a elas a fim de oferecer o melhor cuidado possível a gestante. A capacitação e 
atualização deve ser uma estratégia/ferramenta contínua dentro da equipe para que o profissional se torne apto a acompanhar esse ritmo de mudanças, expandindo e atualizando conhecimentos. ${ }^{16-17}$

\section{Conclusão}

Fica evidente através dos dados da pesquisa, que os enfermeiros tem dificuldades para realização do prénatal de baixo risco nas Estratégias de Saúde da Família. Com isso, pode-se perceber que há uma necessidade de criar e implementar estratégias para assegurar uma linha de cuidados a gestante, para que o Pré-Natal seja efetivo e adequado de acordo com as recomendações do Ministério da Saúde.

A melhoria da assistência pré-natal não está pautada apenas no atendimento clínico em si, mas na integração entre os diferentes serviços de saúde necessários à mãe ao neonato e ao pai, vinculados em Redes de Atenção à Saúde, assegurando o acesso oportuno a serviços de qualidade.

É evidente no estudo, que os enfermeiros passam por várias dificuldades, e que realizar um pré-natal adequado é um grande desafio. De acordo com os dados da pesquisa, uma das adversidades encontra-se no próprio profissional enfermeiro, pois foi constatado em alguns profissionais, a falta de prestatividade, fragilidades para realizar o acolhimento e procedimentos que são rotina do pré-natal, essa adversidade contribui negativamente para a adesão das mulheres ao Pré-Natal, tornando a consulta insatisfatória e afastando as mesmas do ambiente de cuidado, fica claro a necessidade da capacitação do profissional e mudanças de postura no atendimento a população. A precariedade dos recursos físicos, humanos e materiais também é algo que interfere a assistência a gestante, pois na maioria das vezes o local de atendimento e para as atividades em grupo é inapropriado interferindo também na qualidade da consulta e na privacidade da gestante.

O desconhecimento da população quanto as atribuições, habilidades e competências do profissional de enfermagem, é outro entrave, que se torna uma dificuldade para adesão das gestantes a consulta de pré-natal realizada pelo o enfermeiro. Ficando claro a preferência das mulheres para a consulta com o profissional médico. As competências do enfermeiro precisam ser mais trabalhadas e divulgadas na comunidade, para que de fato a população possa ter o conhecimento, consciência das atribuições, segurança e valorização da profissão.

Algumas estratégias para uma melhor assistência no Pré-Natal como a busca ativa das gestantes para que haja identificação precoce de situações de risco, acompanhamento e fortalecimento do vínculo criado entre a equipe e a gestante, o grupo de gestante para orientar e sanar as dúvidas das gestante, possibilitando o intercâmbio de experiências e conhecimentos entre elas, $o$ acolhimento adequado, com atendimento humanizado, fazendo-as se sentirem seguras, e por fim, a capacitação e atualização dos profissionais para prestar um cuidado mais qualificado.

Portanto, o estudo mostrou-se pertinente para a enfermagem visto que, além de identificar desafios/ dificuldades enfrentados pelos enfermeiros na realização do Pré-Natal na Estratégia de Saúde da Família e estratégias para melhorar a assistência a gestante, proporciona a reflexão sobre a prática profissional do enfermeiro a respeito do cuidado prestado a gestante. Pois é necessário que os mesmos tenham consciência do seu papel nesse momento assistencial e que procurem por melhorias tanto individualmente de acordo com suas funções como em conjunto para que realmente aconteça as mudanças esperadas e com isso o Pré-Natal seja satisfatório e de qualidade

\section{Referências Bibliográficas}

1. Serrazina MF, da Silva GSV. Captação da Gestante para Pré-Natal Precoce. Revista Pró-UniverSUS. 2019 Jan./Jun.; 10 (1): 29-34.

2. Brasil, Ministério da Saúde (BR) Secretaria de Atenção à Saúde Departamento de Ações Programáticas Estratégicas. Manual Técnico PréNatal e Puerpério: Atenção Qualificada e Humanizada. Série Direitos Sexuais e Direitos Reprodutivos - Caderno n ${ }^{\circ}$ 5. Brasília (DF): Ministério da Saúde; 2006.

3. Brasil, Ministério da Saúde. Estratégia Saúde da Família. Secretaria de Atenção Primária à Saúde. Disponível em: https://www.saude.gov.br/ acoes-e-programas/saude-da-familia/implantacao-da-estrategia. Acesso em 5 de dezembro de 2020.

4. $\quad$ Brasil, Lei $n^{\circ} 7.498$, de 25 de junho de 1986 (BR). Dispõe sobre a Regulamentação do Exercício da Enfermagem e dá outras providências. Diário Oficial da União. Brasília (DF), de 26 de junho de 1986. Seção I fls. 9.273 a 9.275. Disponível em: http://www.planalto.gov.br/ccivil_03/leis/ 17498.htm

5. Nunes ADS, Amador AE, Dantas APQM, Azevedo UM, Barbosa IR. Acesso à assistência pré-natal no Brasil: Análise dos dados da pesquisa nacional de saúde. Rev. Bras. Promoção da Saúde. 2017 Jul./Set; 30 (3): $1-10$.

6. Gil AC. Métodos e técnicas de pesquisa social. 6. ed. São Paulo: Atlas, 2008 .

7. Lakatos EM, Marconi MA. Fundamentos de metodologia científica. 5. ed. São Paulo: Atlas, 2003

8. Mynaio MCS, Deslandes SF, Neto OC, Gomes R. Pesquisa Social. Teoria, método e criatividade. Petrópolis, Rio de Janeiro: $21^{\mathrm{a}}$ Ed. Vozes, 2002.

9. Gomes CBA, Dias RS, Silva WGB, Pacheco MAB, Sousa FGM, Loyola CMD. Consulta de Enfermagem no Pré-Natal: Narrativas de Gestantes e Enfermeiras. Texto Contexto Enfermagem. 2019.

10. Silva CS, Souza KV, Alves VH, Cabrita BAC, Silva LR. Atuação do Enfermeiro na Consulta Pré-Natal: Limites e Potencialidades. J. res. fundam. care. 2016. Abr./Jun. 8(2):4087-4098.

11. Leal NJ, Barreiro MSC, Mendes RB, Freitas CKAC. Assistência ao Pré-Natal: Depoimento de Enfermeiras. J. res.: fundam. care. 2018. Jan./mar. 10(1): 113-122.

12. Silva AA, Jardim MJA, Rios CTF, Fonseca LMB, Coimbra LC. PréNatal da Gestante de Risco Habitual: Potencialidades e Fragilidades. Rev. Enferm. UFSM, Santa Maria, v.9, ex, p. 1-20, 2019. 
13. Sousa PHSF, Azevedo MVC, Torres RC, Azevedo WMSM, Souza GG. Desafios do Enfermeiro no Pré-Natal de Baixo Risco. Journal Of Health Connections, v.9, n.2. p.77-90.

14. Sehnem GD, Saldanha LS, Arboit J, Ribeiro AC, Paula FM. (2019). Consulta de Pré-Natal na Atenção Primária à Saúde: Fragilidades e Potencialidades da Intervenção de Enfermeiros Brasileiros. Revista de Enfermagem Referência, 5(1), e19050.

15. Castro LS, Rached CD. Acolhimento Humanizado no Cuidado PréNatal as Gestantes da ESF. International Journal Of Health Management. Ed.2. 2019.

16. Costa CSC, Vila VSC, Rodrigues FM, Martins CA, Pinho LMO. Características do Atendimento Pré-Natal na Rede Básica de Saúde. Rev. Eletr. Enf. 2013 abr/jun;15(2):516-22.

17. Silva EP, Lima RT, Osório MM. Impacto de estratégias Educacionais no Pré-Natal de Baixo Risco: Revisão sistemática de Ensaios Clínicos Randomizados. Ciência \& Saúde Coletiva, 21(9):2935-2948, 2016. 